\title{
A Critical Review of Outcomes of Cancer during the COVID-19 Pandemic
}

\begin{abstract}
The recent outbreak of COVID-19 has created an alarming fear, apprehension, and worry world over. Cancer patients represent a subgroup that is vulnerable and is under high risk. It is, therefore, necessary to analyze factors that predict outcomes in these patients so that they can be triaged accordingly in order to mitigate the effects of COVID-19 on cancer management. To determine the factors affecting cancer patients in COVID-19. A systematic search was performed to identify all relevant studies on PubMed, Embase, and Google Scholar published until April 5, 2020. Relevant articles that reported the incidence, demographic and clinical characteristics, treatment, and outcomes of cancer patients infected by COVID-19 were included in the analysis. Among 559 articles that were further screened, 14 articles fulfilled the inclusion criteria. The incidence of cancer across various studies ranged from $0.5 \%$ to $2.43 \%$. Males were more than females, and the mean age affected was $63.1-66$ years. Lung cancer was the most common subtype $(25 \%-58.3 \%)$. Cancer patients, as reported, had a higher risk of progressing to severe events (hazard ratio:3.56, $95 \%$ confidence interval: $1.65-7.69 ; P<0.0001)$. Nearly $39 \%-53.6 \%$ of patients who had a recent history of anticancer therapy developed severe events. Individuals with cancer feared the risk of complications. Cancer patients have worse outcomes from COVID-19, compared to the general population, providing a reason to pay more timely attention. High-risk patients should have vigorous screening and intensive surveillance. Anticancer treatment during COVID-19 should be modified based on the type and prognosis of cancer.
\end{abstract}

Keywords: Cancer, COVID-19, malignancy, severe acute respiratory syndrome-related coronavirus

\section{Introduction}

Severe acute respiratory syndrome-related coronaviruses (SARS-CoVs) are singlestranded, positive-sense, nonsegmented, enveloped RNA viruses. In the past, the world has seen three other "novel" CoV outbreaks that caused substantial destruction. The recent outbreak of COVID-19 has created an alarming fear, apprehension, and worry world over. With the number of confirmed cases on the rise, governments and health-care workers are faced with the challenge of curbing its spread and the resultant damage.

The first pandemic of the $21^{\text {st }}$ century was caused by the SARS-CoV-1. ${ }^{[1]}$ It presented as atypical pneumonia in the latter half of $2002^{[2,3]}$ in Foshan, Guangdong Province, mainland China, from where it spread to Hong Kong, ${ }^{[4]}$ Vietnam, Canada, and Singapore. ${ }^{[5]}$ The epidemic lasted about a year infecting over 8000 individuals and resulting in 774 deaths with a case fatality rate of $9.5 \%{ }^{[6]}$

This is an open access journal, and articles are distributed under the terms of the Creative Commons Attribution-NonCommercial-ShareAlike 4.0 License, which allows others to remix, tweak, and build upon the work non-commercially, as long as appropriate credit is given and the new creations are licensed under the identical terms.

For reprints contact: WKHLRPMedknow_reprints@wolterskluwer.com
A decade later, another $\mathrm{CoV}$ emerged in the Middle East called the Middle East respiratory syndrome-CoV (MERS-CoV). MERS-CoV was a zoonotic virus that entered into the human lineage from dromedary camels in the Arabian Peninsula. ${ }^{[7]}$ In comparison to SARS-CoV, MERS-CoV had a much higher case fatality rate of around $35 \%$, but it has allowed control due to low $R 0$ (basic reproduction number) ${ }^{[6]}$ It claimed around 858 lives with 2494 laboratory-confirmed cases.

In December 2019, reports emerged of a cluster of cases in Wuhan, China, ailing with pneumonia of unknown etiology. In the following week, a strain of $\mathrm{CoV}$ was isolated from these patients. It was initially referred to as novel CoV 2019 but was officially named by the World Health Organization (WHO) on 11 February 2020 as COVID19. The incubation period of the virus ranges from 2 to 14 days (median 5 days). Angiotensin-converting enzyme-2 (ACE2) has been found as the receptor through which the virus invades the respiratory mucosa. ${ }^{[8]}$

How to cite this article: Sharin F, Singh AG, Qayyumi B, Chaturvedi P. A critical review of outcomes of cancer during the COVID-19 pandemic. Indian J Med Paediatr Oncol 2020;41:461-7.
Florida Sharin, Arjun G Singh, Burhanuddin Qayyumi, Pankaj Chaturvedi

Department of Head and Neck Oncology, Tata Memorial Center and HBNI, Mumbai, Maharashtra, India

Submitted: 24-Apr-2020 Revised: 24-Jun-2020 Accepted: 25-Jun-2020 Published: 29-Aug-2020

Address for correspondence: Dr. Pankaj Chaturvedi, Department of Head and Neck Oncology, Tata Memorial Centre, Mumbai, Maharashtra, India.

E-mail: chaturvedi.pankaj@ gmail.com

Access this article online Website: www.ijmpo.org DOI: 10.4103/ijmpo.ijmpo_187_20 Quick Response Code:

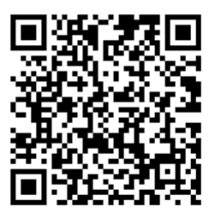


In the last century, the world has faced pandemics such as the plague; smallpox; cholera; yellow fever; and Spanish-, Russian-, Hong Kong- and Asian-flu that claimed millions of lives. The H1N1 swine flu pandemic which originated in Mexico in 2009 spread across the rest of the world infecting roughly 1.4 billion people and killing between 151,700 and 575,400 according to the Centers for Disease Control and Prevention (CDC). Ebola spread across Guinea, Liberia, and Sierra Leone between 2014 and 2016 reporting 28,610 cases and 11,308 deaths.

Cancer patients receive special consideration as they represent a subgroup that is immunocompromised and is under high risk. While coping up with the fears associated with the diagnosis of cancer, the COVID-19 pandemic places another hurdle in their path. It is, therefore, necessary to analyze factors that predict outcomes in these patients so that they can be treated effectively.

\section{Methodology}

A systematic search was performed to identify all relevant studies on PubMed, Embase, and Google Scholar published until April 5, 2020, as per the PRISMA guidelines. The following MESH terms were used: Beta coronavirus or severe acute respiratory syndrome coronavirus 2 or COVID-19 or Coronavirus Infections or Coronavirus and Neoplasms or Cancer Care Facilities or Cancer Survivors or Radiation Oncology or Drug Therapy or Chemotherapy, Adjuvant or Chemotherapy, Cancer, Regional Perfusion or Induction Chemotherapy or Maintenance Chemotherapy. Additional searches were done from reference lists of included studies to avoid any missing articles.

Relevant articles that reported the incidence, demographic and clinical characteristics, treatment, and outcomes of cancer patients infected by COVID-19 were included in the analysis. Articles were graded as per the level of evidence they reported using the Oxford Centre for Evidence-Based Medicine system. The search resulted in 559 articles that were further screened according to the inclusion and exclusion criteria. Articles based on influenza and SARS were excluded, which resulted in 14 articles which were dealt with cancer during the COVID-19 pandemic, however demographic data were available only in three studies. As the sample size was less with limited data available from the quoted studies, statistical analysis was not done.

\section{Results}

The search resulted in 559 articles that were further screened by the authors. Out of these, 14 articles fulfilled the inclusion criteria.

\section{Demographics}

The demographic details about the cancer patients in various studies are tabulated in Table 1.

A nationwide analysis in China by Liang et al. ${ }^{[10]}$ found that cancer patients were of advanced age and more likely to be associated with a history of smoking, severe baseline computed tomography (CT) manifestation, and a higher risk of progressing to severe events (hazard ratio [HR]: 3.56, 95\% confidence interval [CI] [1.65-7.69]; $P<0.0001)$.

The most common symptom on admission in Zhang et al.'s study was fever $(n=23,82.1 \%)$, followed by cough $(n=22,81 \%)$ and fatigue $(n=18,64.3 \%)$. Blood parameters were suggestive of anemia in $21(75 \%)$, lymphopenia in $23(82.1 \%)$, and leukopenia in $9(32.1 \%)$ patients. Levels of albumin were on the lower side in $25(89.3 \%)$ patients, and $92.9 \%$ of the patients had normal prolactin levels. Levels of serum globulin (11 [39.3\%]), lactate dehydrogenase (10 [50\%]), C-reactive protein levels (23 [82.1\%]), erythrocyte sedimentation rate (16 [57.1\%]), and D-Dimer (11 [39.3\%]) were found to be elevated. Chest CT on admission was abnormal in the entire cohort.

Higher dose of corticosteroids was administered for longer duration in patients with severe events, but it failed to demonstrate any statistically significant difference (dose [mg/kg/day]: 1.0 vs. 0.6, period [day]: 3.0 [2.0-4.8] vs. 5.0).

A significant number of severe cases required mechanical ventilation (noninvasive: $53.3 \%$ vs. $0 \%, P<0.001$ ) as compared to nonsevere intensive care unit (ICU) cases (noninvasive: $13.3 \%$ vs. $0 \%, P<0.001$ ). The median period of mechanical ventilation for noninvasive ventilation was 2.5 days (1.0-5.0 days) and 2.5 days for invasive cases.

\begin{tabular}{|c|c|c|c|}
\hline Parameters & Zhang et al..${ }^{[9]} n(\%)$ & Liang et al.,${ }^{[10]} n(\%)$ & Yu et al. ${ }^{[11]} n(\%)$ \\
\hline $\mathrm{N}$ & 28 & 18 & 12 \\
\hline Male:female & $17(60.7): 11(39.28)$ & $12(66.7): 6(33.3)$ & $10(83.33): 2(16.7)$ \\
\hline Mean age (years) & 65 & 63.1 & 66 \\
\hline Comorbidity & $11(39.28)$ & $22.2 \%$ & - \\
\hline Diabetes & $4(14.3)$ & $2(11.11)$ & - \\
\hline $\begin{array}{l}\text { Chronic cardiovascular and cerebrovascular disease } \\
\text { (including hypertension and CHD) }\end{array}$ & $4(14.3)$ & $3(16.7)$ & - \\
\hline Chronic pulmonary disease (including COPD and asthma) & $1(1.36)$ & $1(5.5)$ & - \\
\hline Chronic liver disease (including chronic hepatitis B and cirrhosis) & $2(7.1)$ & - & - \\
\hline Chronic renal disease & 1 & $1(5.5)$ & - \\
\hline
\end{tabular}

CHD: Coronary heart disease, COPD: Chronic obstructive pulmonary disease 


\section{Incidence of cancer among COVID-19 patients}

Eight studies have specified the number of cancer patients being infected in the COVID-19 pandemic. The results are summarized in Table 2 .

Single-institutional studies from China like that of Huang et al., ${ }^{[12]}$ Chen et al.., ${ }^{[13]}$ Wang et al.., ${ }^{[14]}$ and $\mathrm{Wu}$ et al. ${ }^{[16]}$ have reported the incidence of cancer among the various other COVID-19-positive cases.

\section{Distribution of various cancers}

The studies which looked into the distribution of various cancers among the COVID-19 patients are summarized in Table 3 .

Among the several sites of cancer in the study by Zhang et al. ${ }^{[9]}$ lung $(25 \%, n=7)$ was the most common site, and it was observed that symptoms of dyspnea occurred much earlier in cancer patients compared to that in the general population $(1.0[0.0-3.5] \text { vs. } 8.0 \text { [5.0-13.0] days })^{[19]}$ and other cancer patients (1.0 [0.0-3.5] vs. 5.0 [4.0-7.0] days).

Lung was also the most frequent site $(28 \% n=5)$ in the study by Liang et al., ${ }^{[10]}$ however, compared to other sites, patients with lung cancer $(1 / 5,20 \%$ vs. $8 / 13,62 \%)$ did not have much likelihood of developing severe events.

\section{Treatment history}

The treatment protocol among the various studies was according to their institutional protocols. Detailed treatment history was not available in all the studies. Table 4

\begin{tabular}{lccc}
\hline \multicolumn{4}{c}{ Table 2: Incidence of cancer among COVID-19-positive } \\
patients
\end{tabular}

summarizes the antitumoral treatment history received by patients across the various studies.

Staging of the tumor was presented only in the study by Zhang et al. ${ }^{[9]}$ where $18(64.3 \%)$ patients were in Stages I-III and $10(35.7 \%)$ patients in Stage IV disease. On descriptive analysis, it was found that $70 \%(7 / 10)$ of Stage IV cancer patients had developed severe events, whereas only $44.4 \%$ of the rest had such events with no statistically significant association on univariate analysis. The tumor stage could not be included in multivariate cox model analysis due to a high correlation between stage and the antitumor treatment within 14 days (correlation coefficient $r=-0.518, P=0.005)$.

In the study by Zhang et al., ${ }^{[9]}$ the patients receiving antitumoral treatment within 2 weeks, 5 (83\%), developed severe events.

Multivariate analysis adjusted for gender and age revealed that those patients who received antitumor treatment within 2 weeks before COVID-19 infection had a greater risk of developing severe events with marginal statistical significance (HR: 4.079, 95\% CI: 1.086-15.322; $P=0.037)$.

Nearly $75 \%$ of the patients (3/4) who underwent surgery or chemotherapy in the previous month had a high risk of developing clinically severe events than those $(6 / 14)$ not receiving chemotherapy or surgery in the study by Liang et $a l .^{[10]}$ It was additionally confirmed by logistic regression (odds ratio [OR]: 5.34, 95\% CI: 1.80-16.18; $P=0.0026$ ) after adjusting for other risk factors such as smoking, age, and other comorbidities.

In the study by $\mathrm{Yu}$ et al., ${ }^{[1]}$ fewer than half of these COVID-19 patients were undergoing active treatment for malignancy.

\section{Morbidity and mortality in cancer patients}

According to the study by Zhang et al., ${ }^{[9]}$ out of the 28 cancer patients, $53.6 \%$ of the patients advanced to severe events, $21.4 \%$ required ICU admission, $35.7 \%$ had life-threatening complications, and $28.6 \%$ of the patients succumbed to death. The cause of death encompassed

\begin{tabular}{|c|c|c|c|}
\hline Types of cancer & Zhang et al. $.^{[9]}(n=28), n(\%)$ & Liang et al. ${ }^{[10]}(n=18), n(\%)$ & Yu et al. ${ }^{[11]}(n=12), n(\%)$ \\
\hline Lung cancer & $7(25.0)$ & $5(28)$ & $7(58.3)$ \\
\hline Esophagus cancer & $4(14.3)$ & - & - \\
\hline Breast cancer & $3(10.7)$ & $3(16.6)$ & $1(8.3)$ \\
\hline Larynx & $2(7.1)$ & - & - \\
\hline Liver cancer & $2(7.1)$ & - & - \\
\hline Prostatic cancer & $2(7.1)$ & - & - \\
\hline Cervical cancer & $1(3.6)$ & - & - \\
\hline Gastric cancer & $1(3.6)$ & - & - \\
\hline Colorectal cancers & $2(7.1)$ & $4(22.2)$ & $2(16.7)$ \\
\hline Adrenal neoplasm & - & $1(5.5)$ & - \\
\hline
\end{tabular}




\begin{tabular}{|c|c|c|c|}
\hline \multicolumn{4}{|c|}{ Table 4: Antitumoral treatment details of COVID-19-positive cancer patients } \\
\hline Treatment & Zhang et al. $.^{[9]}(n=28), n(\%)$ & Liang et al. ${ }^{[10]}(n=18), n(\%)$ & Yu et al. ${ }^{[11]}(n=12), n(\%)$ \\
\hline Surgery & $21(75.0)$ & $13(72.2)$ & $1(8.3)$ \\
\hline Chemo/radiotherapy & $25(89.3)$ & $9(50)$ & $7(58.3)$ \\
\hline Target/immunotherapy & $6(21.4$ & $3(16.7)$ & $1(8.3)$ \\
\hline Best supportive care & - & - & $4(33.3)$ \\
\hline Details not available & - & $1(5.6)$ & $1(8.3)$ \\
\hline
\end{tabular}

acute respiratory distress syndrome (ARDS) in about $62.5 \%(5 / 8)$, pulmonary embolism in $12.5 \%$ (1/8), septic shock in $12.5 \%(1 / 8)$, and acute myocardial infection in $12.5 \%(1 / 8)$ of patients.

On multivariate analysis for the risk of developing severe events, there was no statistically significant correlation with age (HR: $1.455 ; 95 \%$ CI: $0.478-4.430 ; P=0.509$ ) and gender (HR: 0.574; 95\% CI: 0.162-2.038; $P=0.390$ ).

Liang et al. ${ }^{[10]}$ used Cox regression model to estimate the time-dependent hazards of progressing to severe events, and it was seen that cancer patients deteriorated more rapidly (median time to severe event - 13 days) (interquartile range: 6-15) than those without cancers (median time to severe event - 43 days) (20 - not reached), $P<0$. 0001, HR: 3.56, 95\% CI: 1. 65-7.69, after adjusting for age.

The only risk factor for developing severe events in the study by Liang et al. ${ }^{[10]}$ was found to be old age (OR: 1.43, 95\% CI: 0.97-2.12; $P=0.072$ ). While comorbidities such as hypertension (OR: 1.878, 95\% CI: 1.217-2.898; $P=0.004)$, chronic obstructive pulmonary disease (COPD) (OR: 3.397, 95\% CI: 1.373-8.409; $P=0.008$ ), and diabetes mellitus (OR: 2.206, 95\% CI: 1.331-3.656; $P=0.002$ ) were not associated with the risk of developing severe events.

In a case series by Grasselli et al., ${ }^{[19]}$ the demographic details of COVID-19 patients admitted to ICUs in Lombardy, Italy, were gathered telephonically by the ICU network team. Data were collected from February to March 18, 2020. Among 1591 patients requiring admission into ICU, 81 had a history of malignancy either in active or remission stage, with 69 of those patients above 60 years.

In a large case series of 72,314 patients published by the Chinese $\mathrm{CDC},{ }^{[18]}$ the overall case fatality was around $2.3 \%$ and in patients with a history of malignancy, it was $5.6 \%$.

The WHO China Joint Mission on COVID-19 reported a case fatality rate of $7.6 \%$ for cancer.

Onder et al. ${ }^{[20]}$ analyzed a subsample of 355 patients who died due to COVID-19 in Italy. The mean age was found to be 79.5 years (standard deviation 8.1) and 72 patients died due to cancer, leading to a case fatality rate of $20.3 \%$.

\section{Biological basis of susceptibility}

Cancer patients are more susceptible to infection due to their immunocompromised status owing to multiple reasons such as the disease itself, malnutrition, postsurgical status, or the anticancer treatment that they receive.

In a review article by Galluzzi et al., ${ }^{[21]}$ they have reported that as tumors evolve, they favor the release of myeloid-derived suppressor cells (MDSCs) from the bone marrow, which exerts immunosuppressive effects both intratumorally and systemically. In addition, myelosuppression due to cytotoxic chemotherapy or early phase of hematopoietic stem cell transfer, conventional chemotherapy, and targeted therapy drugs such as rituximab may alter both humoral and T-cell-mediated immunity, making the individual more susceptible to COVID-19.

$\mathrm{Cai}^{[22]}$ analyzed transcriptomic databases of lung tissue and found that smoking tobacco increases the gene expression of ACE2 in the lung, which is also the binding receptor for COVID-19. This could explain the reason for smokers being susceptible to COVID-19 as they both share the same receptor.

Malnutrition in a cancer patient can be due to multiple reasons such as nausea and vomiting because of chemotherapeutic drugs, loss of appetite, the morbidity associated with surgery, or long-term hospitalization. Moreover, $20 \%-80 \%$ of cancer patients become malnourished during their clinical course. ${ }^{[23-25]}$ This can adversely affect the immunity of a patient, making him/her more prone to infection by respiratory pathogens.

In the study by Zhang et al., ${ }^{[9]}$ lung cancer patients showed reduced lung volume due to tumor co-existing with features of pneumonia.

\section{Quality of life}

Quality-of-life assessment is a multidimensional paradigm measuring subjective assessment of well-being and health status including both mental and physical health. A qualitative survey was done by Casanova et al. ${ }^{[26]}$ in the Milan focal point of Italy on young cancer patients during COVID-19 in order to assess their level of stress. According to the survey, it was found that a relatively large proportion of these patients felt themselves to be at risk of severe complications. A semi-structured questionnaire was developed by a committee consisting of Youth Project's staff (psychologists and physicians) and was later approved 
by the ethics committee. Questionnaires were given to patients receiving treatment, on follow-up, and healthy peers. It was seen that a large proportion of patients who were receiving cancer treatment and on follow-up were worried about severe complications compared to healthy peers.

\section{Discussion}

COVID-19 has spread rampantly across the globe originating from China. Patients with cancers are undoubtedly affected severely due to the ongoing pandemic. The incidence of cancer reported across various studies varies from $0.5 \%$ to $2.43 \%$. The incidence of cancer was higher than the overall incidence among the Chinese population $(0.29 \%)$ according to cancer epidemiology statistics 2015. ${ }^{[27]}$ However, the following points should be considered:

1. Duration of the study was short, studies were conducted during the epidemic, and many were single-institutional studies

2. Suspected or undiagnosed cases were not included

3. The data available were quite heterogeneous, and many of the studies are only from China, especially from the area when the epidemic started, and hence cannot be extrapolated to the population in other parts of the world

4. Immunocompromised cancer patients are more likely to acquire infections.

Among the retrospective studies exclusively analyzing COVID-19-positive cancer patients, it was observed that lung cancer was common overall. Studies by Zhang et $a l . .^{[9]}$ and Liang et al. ${ }^{[10]}$ were multicentric studies where the incidence of lung cancer was more or less similar.

Extensive prospective epidemiologic data have clearly established cigarette smoking as the major cause of lung cancer. ${ }^{[28]}$ Approximately $50 \%$ of ARDS can be directly attributed to smoking tobacco. ${ }^{[29]}$ Smoking increases the expression of ACE2 in the lungs, which is a binding site for COVID-19. ${ }^{[30]}$ According to a recent meta-analysis by Emami et al., ${ }^{[31]}$ the pooled prevalence of COVID-19 patients admitted in hospitals with a history of tobacco smoking was estimated to be around $7.63 \%$. Smokers were observed to be more susceptible to COVID-19 infection.

Previous studies on MERS-CoV-2 have demonstrated dipeptidyl peptidase IV to be a specific receptor for the virus, and it is upregulated in COPD patients and smokers, making them more vulnerable to this infection. ${ }^{[32]}$

Patients with lung cancer, with poor baseline pulmonary function and endurance, were more likely to develop more severe anoxia and deteriorate more rapidly with COVID-19. ${ }^{[9]}$ Lung injury can occur due to primary lung tumors, metastasis, or radiation pneumonitis. These can explain the reason why lung cancer patients are the ones who are affected by COVID-19 on a larger scale.
Smoking can lead to lung cancer and COPD and increase susceptibility to acquiring COVID-19. Although smoking was predominantly more among cancer groups in the study by Liang et al., ${ }^{[10]}$ it failed to establish any statistical significance.

Males were more than females among all studies with mean age ranging from 63 to 66 years, implying elderly age group being affected. Elderly individuals had the risk of progressing to severe events.

Cardiovascular diseases and diabetes were among the most prevalent comorbidities. A systematic review and meta-analysis by Emami et al. ${ }^{[31]}$ has summarized that cardiovascular diseases, hypertension, COPD, and chronic kidney disease were among the most prevalent underlying comorbidities in hospitalized COVID-19 patients, which is similar to our findings in cancer patients.

Metabolic syndrome-related health conditions such as hypertension, cardiovascular diseases, and diabetes are known to downregulate innate immunity in the host. However, in the study by Liang et al., ${ }^{[10]}$ progression to severe events was not related to comorbidities. Given that COVID-19 has a relatively protracted incubation period and during this phase, the infected person can transmit the virus to another without being symptomatic, it is strongly recommended that elderly cancer patients with chronic or underlying diseases avoid close contact with others in the community, especially in areas where the epidemic is rampant.

Findings of ground-glass opacity and patchy consolidation on CT scan of cancer patients were similar to overall COVID-19 infected patients. ${ }^{[33]}$ The role of systemic steroids is controversial in viral pneumonia, as it is thought to be an immunosuppressant. However, it failed to demonstrate any benefit in reducing the incidence of severe events. ${ }^{[9]}$

In their study, cancer patients who had ongoing treatment or those who had completed treatment within the past month were at risk of developing clinical severe events such as ICU admissions, mechanical ventilation, or death. Zhang et al. ${ }^{[9]}$ reported $53.6 \%$ of cancer patients developing severe events, whereas Liang et al. ${ }^{[10]}$ had only $39 \%$ of people developing severe events. This discrepancy is due to the following reasons:

1. The definition of severe events by both groups was different: Zhang et al. ${ }^{[9]}$ included ICU admission or mechanical ventilation or death, whereas Liang et al. ${ }^{[10]}$ defined it as ICU admission or invasive ventilation or death, narrowing the cohort to only those requiring invasive ventilation

2. Zhang et al..$^{[9]}$ included cases from Wuhan, whereas Liang et $a .^{[10]}$ included patients from the entire nation. Wuhan, on the other hand, had to face a dire shortage of resources due to the sudden outbreak and delay in delivering health facilities, leading to an increase in mortality.

The study by Grasselli et al. ${ }^{[19]}$ had many limitations as newly setup ICUs were not included, it was telephonic data 
collection, and the follow-up period was relatively short and hence, the outcomes and mortality may change.

Death was seen in $28.6 \%$ of the patients who developed critical events in the study by Zhang et al. ${ }^{[9]}$ In comparison to the general COVID-19 population, 5\% reached clinically critical status, and the case fatality was nearly half $(49 \%) .{ }^{[18]}$ Studies from China reported a case fatality of 5.6\%-7.6\%, whereas Onder et al. ${ }^{[20]}$ from Italy reported a case fatality rate of $20.3 \%$. It is also to be considered that a large proportion of people were aged 90 or above in Italy, and this group has a very high case fatality rate $(22.7 \%)$.

Zhang et $a l .{ }^{[9]}$ reported that $28.6 \%$ of patients developed COVID-19 infection during hospital admission suspecting nosocomial transmission. Nosocomial transmission is reported by Wang et al..$^{[14]}$ to be around $41 \%$. Cancer patients can acquire infections in hospitals during their course of anticancer treatment. This emphasizes vigorous screening and isolation of suspected patients at cancer centers. Precautionary measures should also extend to the family members and the health-care personnel attending such patients to prevent infection and transmission. The European Society for Medical Oncology recommends a tiered approach according to the priority of intervention required, and also the benefit/risk ratio, prognosis, patient preferences, and comorbidities should be taken into consideration.

While navigating through all these, an epidemic like COVID-19 has made their condition even worse as the medical facilities are likely to be jeopardized due to the overwhelming patients. Life in a pandemic is more disturbing due to social distancing, isolation, lockdown, and fear of contracting infections, which lead to a cycle of concern, worry, and mental agony. There are also groundless fears due to the fabricated news and information circulated in social media. Delays in treatment may lead to fear of death, progression, or recurrence in cancer patients.

Thus, the following points have to be borne in mind while managing cancer patients:

1. At present, there is no direct evidence to support withholding anti-tumor therapy. Treatment that is likely to worsen the immune status of a patient should be postponed or modified

2. Patients should be triaged according to type and stage of cancer and need for aggressive treatment

3. The possible benefits and risks of infection have to be discussed with the patient and family members. If continuing treatment is the need of the hour, then patients should be guided accordingly

4. The diagnosis of cancer itself has a lot of psychosocial impact on a person. The journey from diagnosis to treatment can affect relationships, finances, and the physical and mental health of a person

5. Telemedicine can help in reaching many patients and decrease anxiety. Cancer patients require follow-ups at regular intervals in order to treat early recurrence, to detect new primaries, or for rehabilitation. Due to lockdown, asymptomatic patients can be followed up telephonically. Any new symptoms or concerns can be dealt, and the necessary solutions can be discussed

6. In certain parts of the country wherein there is no access to oncology facilities, the referral centers can utilize tele-expertize.

Studies have shown that cancer patients have worse outcomes from COVID-19, providing a reason to pay more timely attention to cancer patients in case they deteriorate rapidly. As a result, more intensive surveillance should be considered when cancer patients acquire COVID-19, especially elderly, patients with comorbidities, and patients receiving ongoing treatment. Further prospective studies from different parts of the world with a large sample size are warranted to validate the findings. More robust evidence is required to address various factors such as screening, prevention, quality of life, and treatment modalities.

\section{Conclusion}

Cancer patients with COVID-19 have worse outcomes compared to the general population therefore should undergo vigorous screening and intensive surveillance. Anticancer treatment during COVID19 should be altered according to the type and prognosis of cancer.

\section{Limitations}

1. Due to the recent outbreak of the virus, most of the studies do not have the follow-up data pertaining to the infection, the values of which are crude and may change the mortality pattern

2. The possible benefits and risks of infection have to be discussed with the patient and family members. If continuing treatment is the need of the hour, then patients should be guided accordingly

3. Tumor types were diverse and staging was not included among the various cancers

4. Continued surveillance and accurate reporting of demographics, testing policies, and treatment outcomes is necessary from different places across the globe for better understanding and treatment planning.

Financial support and sponsorship

Nil.

\section{Conflicts of interest}

There are no conflicts of interest.

\section{References}

1. Peiris JS, Yuen KY, Osterhaus AD, Stöhr K. The severe acute respiratory syndrome. The New England J Med 2004;349:2431-41.

2. Zhao Z, Zhang F, Xu M, Huang K, Zhong W, Cai W, et al. Description and clinical treatment of an early outbreak of severe acute respiratory syndrome (SARS) in Guangzhou, PR China. 
J Med Microbiol 2003;52:715-20.

3. Zhong NS, Zheng BJ, Li YM, Poon, Xie ZH, Chan KH, et al. Epidemiology and cause of severe acute respiratory syndrome (SARS) in Guangdong, People's Republic of China, in February, 2003. Lancet (London, England) 2003;362:1353-8.

4. Lee N, Hui D, Wu A, Chan P, Cameron P, Joynt GM, et al. A major outbreak of severe acute respiratory syndrome in Hong Kong. N Engl J Med 2003;348:1986-94.

5. Global surveillance for severe acute respiratory syndrome (SARS). Wkly Epidemiol Rec 2003;78:100-19.

6. Guarner J. Three emerging coronaviruses in two decades: The story of SARS, MERS, and now COVID-19. Am J Clin Pathol 2020;153:420-1.

7. World Health Organization. Middle East Respiratory Syndrome Coronavirus (MERS-CoV). World Health Organization; 2020.

8. Cheng ZJ, Shan J. 2019 Novel coronavirus: Where we are and what we know. Infection 2020;48:155-63. doi:10.1007/ s15010020-01401-y. Available from: https://pubmed.ncbi.nlm.nih. gov/32072569/. [Last accessed on 2020 Jun 24].

9. Zhang L, Zhu F, Xie L, Wang C, Wang J, Chen R, et al. Clinical characteristics of COVID-19-infected cancer patients: A retrospective case study in three hospitals within Wuhan, China. Ann Oncol 2020;31:894-901.

10. Liang W, Guan W, Chen R, Wang W, Li J, Xu K, et al. Cancer patients in SARS-CoV-2 infection: A nationwide analysis in China. Lancet Oncol 2020;21:335-7.

11. Yu J, Ouyang W, Chua MLK, Xie C. SARS-CoV-2 transmission in patients with cancer at a tertiary care hospital in Wuhan, China. JAMA Oncol. Published online March 25, 2020.

12. Huang C, Wang Y, Li X, Ren L, Zhao J, Hu Y, et al. Clinical features of patients infected with 2019 novel coronavirus in Wuhan, China. Lancet 2020;395:497-506.

13. Chen $\mathrm{N}$, Zhou M, Dong $\mathrm{X}, \mathrm{Qu} \mathrm{J}$, Gong $\mathrm{F}$, Han $\mathrm{Y}$, et al. Epidemiological and clinical characteristics of 99 cases of 2019 novel coronavirus pneumonia in Wuhan, China : A descriptive study. Lancet 2020;395:507-13.

14. Wang D, Hu B, Hu C, Zhu F, Liu X, Zhang J, et al. Clinical characteristics of 138 hospitalized patients with 2019 novel coronavirus-infected pneumonia in Wuhan, China. JAMA 2020;323:1061-9.

15. Guan WJ, Ni ZY, Hu Y, Liang WH, Ou CQ, He JX, et al. Clinical characteristics of coronavirus disease 2019 in China. Engl J Med 2020;382:1708-20.

16. Wu J, Liu J, Zhao X, Liu C, Wang W, Wang D, et al. Clinical characteristics of imported cases of COVID-19 in Jiangsu province: A multicenter descriptive study. Clin Infect Dis 2020. doi: 10.1093/cid/ciaa199.

17. Liu K, Fang YY, Deng Y, Liu W, Wang MF, Ma JP, et al. Clinical characteristics of novel coronavirus cases in tertiary hospitals in Hubei Province. Chin Med J (Engl) 2020;133:1025-31.

18. Wu Z, McGoogan JM. Characteristics of and important lessons from the coronavirus disease 2019 (COVID-19) outbreak in China: Summary of a report of 72314 cases from the Chinese Center for Disease Control and Prevention. JAMA 2020;323:1239-42.
19. Grasselli G, Zangrillo A, Zanella A, Antonelli M, Cabrini L, Castelli A, et al. Baseline characteristics and outcomes of 1591 patients infected with SARS-CoV-2 admitted to ICUs of the Lombardy Region, Italy. JAMA 2020;323:1574-81.

20. Onder G, Rezza G, Brusaferro S. Case-fatality rate and characteristics of patients dying in relation to COVID-19 in Italy. JAMA 2020;323:1775-6.

21. Galluzzi L, Buqué A, Kepp O, Zitvogel L, Kroemer G. Immunological effects of conventional chemotherapy and targeted anticancer agents. Cancer Cell 2015;28:690-714.

22. Cai G. Bulk and single-cell transcriptomics identify tobacco-use disparity in lung gene expression of ACE2, the receptor of 2019nCov. MedRxiv 2020.

23. Segura A, Pardo J, Jara C, Zugazabeitia L, Carulla J, de las Peñas $\mathrm{R}$, et al. An epidemiological evaluation of the prevalence of malnutrition in Spanish patients with locally advanced or metastatic cancer. Clin Nutr 2005;24:801-14.

24. Capra S, Ferguson M, Ried K. Cancer: Impact of nutrition intervention outcome-nutrition issues for patients. Nutrition 2001;17:769-72.

25. Ravasco P, Monteiro-Grillo I, Vidal PM, Camilo ME. Nutritional deterioration in cancer: The role of disease and diet. Clin Oncol (R Coll Radiol) 2003;15:443-50.

26. Casanova M, Bagliacca EP, Silva M, Patriarca C, Veneroni L, Clerici CA, et al. How young patients with cancer perceive the Covid-19 (coronavirus) epidemic in Milan, Italy: Is there room for other fears? Pediatr Blood Cancer 2020;19:e28318.

27. Zheng RS, Sun KX, Zhang SW, Zeng HM, Zou XN, Chen R, et al. Report of cancer epidemiology in China, 2015. Zhonghua Zhong Liu Za Zhi 2019;41:19-28.

28. Devesa SS, Grauman DJ, Blot WJ, Fraumeni Jr JF. Cancer surveillance series: Changing geographic patterns of lung cancer mortality in the United States, 1950 through 1994. Journal of the National Cancer Institute 1999;91:1040-50.

29. Iribarren C, Jacobs DR Jr, Sidney S, Gross MD, Eisner MD. Cigarette smoking, alcohol consumption, and risk of ARDS: A 15-year cohort study in a managed care setting. Chest 2000;117:163-8.

30. Xia Y, Jin R, Zhao J, Li W, Shen H. Risk of COVID-19 for patients with cancer. Lancet Oncol 2020;21:e180.

31. Emami A, Javanmardi F, Pirbonyeh N, Akbari A. Prevalence of underlying diseases in hospitalized patients with COVID-19: A systematic review and meta-analysis. Arch Acad Emerg Med 2020;8:e35.

32. Seys LJ, Widagdo W, Verhamme FM, Kleinjan A, Janssens W, Joos GF, et al. DPP4, the Middle East respiratory syndrome coronavirus receptor, is upregulated in lungs of smokers and chronic obstructive pulmonary disease patients. Clinical Infectious Diseases 2018;66:45-53.

33. Shi $\mathrm{H}$, Han $\mathrm{X}$, Jiang $\mathrm{N}$, Cao $\mathrm{Y}$, Alwalid $\mathrm{O}, \mathrm{Gu} \mathrm{J}$, et al. Radiological findings from 81 patients with COVID-19 pneumonia in Wuhan, China: A descriptive study. Lancet Infect Dis 2020;20:425-34. 